\title{
TFE3-Expressing Perivascular Epithelioid Cell Tumor of the Breast
}

\author{
Hyunjin Kim · Jimin Kim \\ Se Kyung Lee ${ }^{1} \cdot$ Eun Yoon Cho \\ Soo Youn Cho \\ Department of Pathology and Translational \\ Genomics, 'Division of Breast Surgery, \\ Department of Surgery, Samsung Medical \\ Center, Sungkyunkwan University School of \\ Medicine, Seoul, Korea \\ Received: June 29, 2018 \\ Revised: August 17, 2018 \\ Accepted: August 30, 2018 \\ Corresponding Author \\ Soo Youn Cho, MD, PhD \\ Department of Pathology and Translational \\ Genomics, Samsung Medical Center, Sungkyunkwan \\ University School of Medicine, 81 Irwon-ro, \\ Gangnam-gu, Seoul 06351, Korea \\ Tel: +82-2-3410-2817 \\ Fax: +82-2-3410-0025 \\ E-mail: sooyoun.cho@samsung.com
}

Perivascular epithelioid cell tumor (PEComa) is a very rare mesenchymal tumor with a distinctive morphology and immunophenotype. PEComas usually harbor TSC2 alterations, although TFE3 translocations, which occur in MiT family translocation renal cell carcinoma and alveolar soft part sarcoma, are also possible. We recently experienced a case of PEComa with TFE3 expression arising in the breast. An 18-year-old female patient presented with a right breast mass. Histologically, the tumor consisted of epithelioid cells with alveolar structure and showed a diffuse strong expression of HMB45 and TFE3. TSC2 was preserved. Melan A and smooth muscle actin were negative. To our knowledge, this is the first Korean case of PEComa of the breast that intriguingly presented with TFE3 expression.

Key Words: Perivascular epithelioid cell neoplasms; TFE3; Breast
Perivascular epithelioid cell (PEC) tumor (PEComa) is a mesenchymal tumor characterized by distinctive histological and immunohistochemical features. PEComas are composed of perivascular epithelioid and spindle cells that are immunoreactive to melanocytic and myogenic markers. These tumors are much more common in females than in males and involve gynecologic as well as other abdominopelvic organs. ${ }^{1}$ PEComas are mostly benign, although some show aggressive behavior. ${ }^{2}$ Not much is known about PEComas mainly due to their rarity, but several recent studies have revealed that a subset of PEComa has TFE3 alterations rather than the more common TSC2/TSC1 abnormalities. ${ }^{3-5}$ We recently encountered a case of PEComa arising in the breast that was TFE3-immunoreactive and predominantly epithelioid in nature. To our knowledge, only one case of breast PEComa has been reported in the English literature, ${ }^{4}$ and the present case is the first to be reported in Korea. Moreover, PEComas with TFE3 gene translocation have been noted in other organs, ${ }^{6}$ but this is the first case involving the breast.

\section{CASE REPORT}

An 18-year-old female presented with a palpable mass in the upper outer quadrant of the right breast. She had no relevant past medical or family history. Ultrasonographic examination revealed that the mass had increased in size from $1.5 \times 1.3 \mathrm{~cm}$ to $3.4 \times 2.7 \mathrm{~cm}$ within a seven-month period. The patient visited Samsung Medical Center in Seoul, Korea, following a biopsy in another hospital, where she was diagnosed with PEComa. Wide excision was performed.

The gross examination showed a well-encapsulated, $4 \times 3.2-$ $\mathrm{cm}-$ sized mass, and the cut surface was homogeneously solid and brown. Microscopic findings revealed a thick fibrous capsule wherein epithelioid cells were arranged in a nested architecture surrounded by branching thin-walled vessels (Fig. 1A). The individual epithelioid cells had clear to granular cytoplasm (Fig. 1B). Spindle cells were not observed. The nuclei of the tumor cells were uniform and round, and showed mild atypia. Occasional melanin pigments were noted in the cytoplasm of the tumor cells (Fig. 1C). Necrosis was not identified, and mitoses were counted at 
up to 3 per 10 high-power fields (HPFs). The tumor showed diffuse strong positivity to HMB45 (1:80, mouse clone HMB45, Dako Corporation, Carpinteria, CA, USA) (Fig. 2A) and TFE3 (1:20, rabbit clone MRQ-37, Cell Marque, Rocklin, CA, USA) (Fig. 2B). TSC2 (1:200, rabbit clone D93F12, Cell Signaling Technology, Danvers, MA, USA) was also positive. Melan A (1:80, mouse clone A103, Dako Corporation), smooth muscle actin (1:100, mouse clone 1A4, Dako Corporation), S100 protein (1:2,000, rabbit polyclonal, Dako Corporation), and cytokeratin (1:500, mouse clone AE1/AE3, Dako Corporation) were not expressed. Moreover, progesterone receptor (PR; 1:800, mouse clone 16, Novocastra Laboratories Ltd., Newcastle upon Tyne, UK) was positive (Fig. 2C), while estrogen receptor (ER; 1:400, mouse clone 6F11, Novocastra Laboratories Ltd.) was negative.

The histologic and immunohistochemical findings were most consistent with PEComa with TFE3 expression.

Ten months after resection, the patient remained healthy with no evidence of recurrence or other unusual findings. This study was approved by the Institutional Review Board of Samsung Medical Center, and the need for informed consent was waived (2018-06-045).

\section{DISCUSSION}

PEComa is a tumor composed of PECs. PECs were first described by Apitz in 1943, who recognized unusual "abnormal myoblasts" in an angiomyolipoma, clear cell sugar tumor (CCST), and lymphangioleiomyomatosis. ${ }^{7}$ Later, these cells were revealed to be immunoreactive for HMB45. The World Health Organization defines PEComa as a "mesenchymal tumor composed of histologically and immunohistochemically distinctive perivascular epithelioid cells."

The most common sites of PEComas are the retroperitoneum, abdominopelvic region, uterus, and gastrointestinal tract. ${ }^{1}$ The typical histologic findings demonstrate a nested architecture of epithelioid and spindle cells with abundant granular eosinophilic or clear cytoplasm. The epithelioid component of these tumors characteristically expresses melanocytic markers, while the spindle cell component occasionally expresses myogenic markers. PEComas occur more commonly in young and middle-aged patients, and the female to male ratio is greater than 6:1.

In the current report, we present a very rare case of PEComa in the breast. The single known previously reported case was that of an extrapulmonary CCST in the breast of a 16-year-old female,
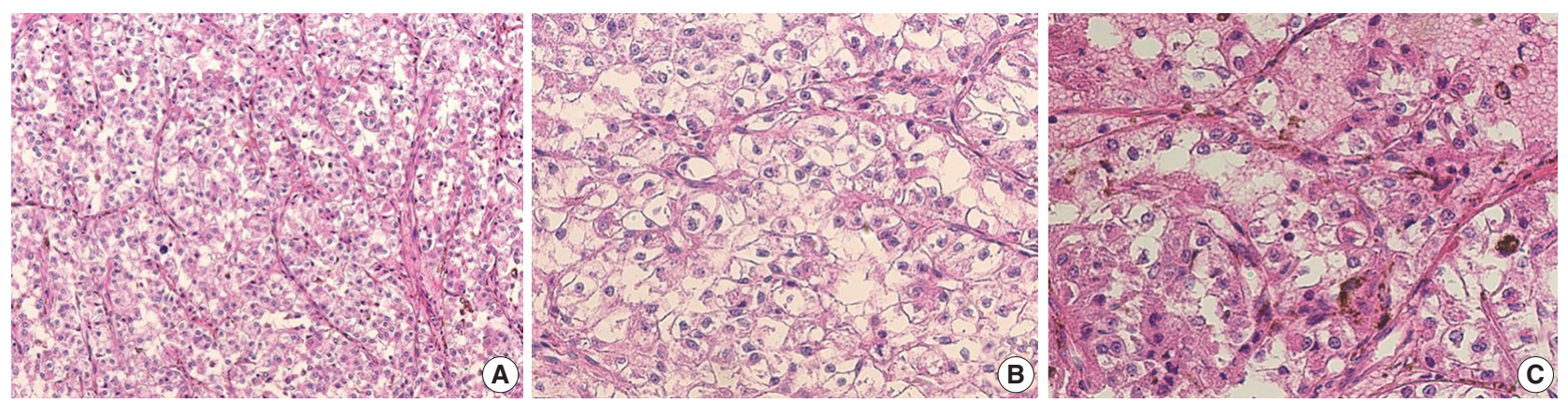

Fig. 1. Microscopic findings of the TFE3-expressing breast perivascular epithelioid cell tumor. (A) Epithelioid cells were arranged in a nested architecture with surrounding branching thin-walled vessels. (B) The individual epithelioid cells show clear to granular cytoplasm. (C) Occasional melanin pigments are noted.

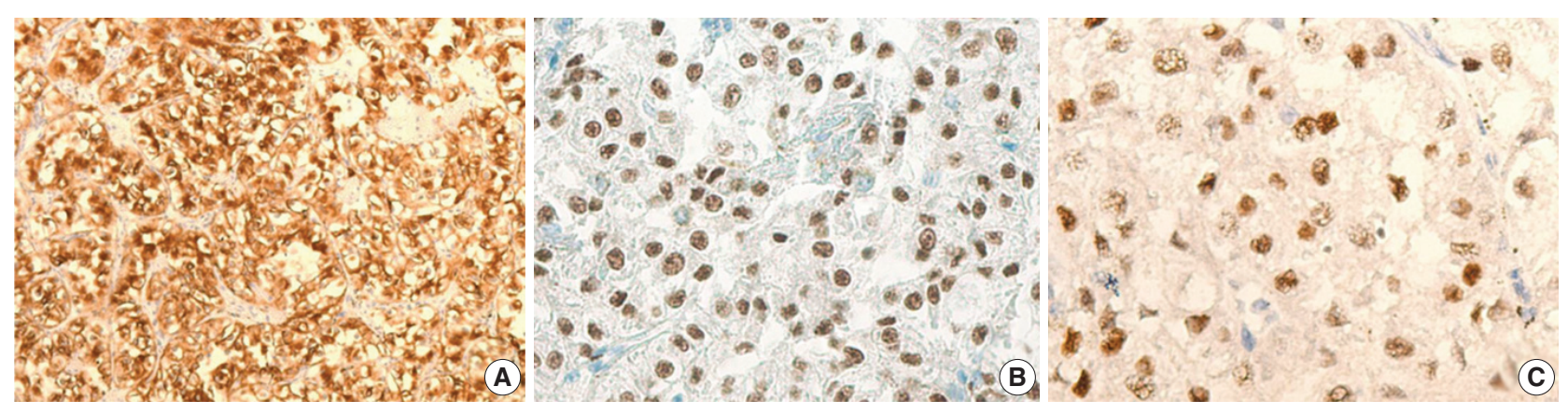

Fig. 2. The immunohistochemical findings of the TFE3-expressing breast perivascular epithelioid cell tumor. The tumor is diffusely positive for human melanoma black 45 (A) and shows nuclear positivity for TFE3 (B) and progesterone receptor (C). 
which showed strong immunohistochemical reactivity to HMB45, melan A, and PR. ${ }^{4}$

In our case, the tumor was positive for HMB45 and PR (Fig. 2A, C) and negative for melan A. Interestingly, this case showed diffuse strong expression of TFE3 (Fig. 2B) with preserved TSC2 staining. The strong positivity of TFE3 was notable. Conventional PEComas, both sporadic and syndromic, frequently shows inactivation of TSC2 or TSC1 and loss of expression in immunohistochemistry. ${ }^{8}$ Recently, Argani $e t$ al. ${ }^{3}$ suggested that there may be a "distinct subset of PEComa" that harbors TFE3 gene fusions. They concluded that such cases tend to present in a "young age group [and] have no association with tuberous sclerosis, with predominant alveolar architecture and epithelioid cytology [and] minimal immunoreactivity for muscle markers," a description that conforms to our case. Schoolmeester $e t$ al. ${ }^{6}$ also reported the distinct morphology and immunohistochemical features of TFE3-rearranged PEComas in the gynecologic tract. TFE3-rearranged PEComas do not share the TSC2/TSC1 genetic abnormalities of conventional PEComas, and retain their protein expressions on immunohistochemistry. ${ }^{7}$ Our case showed strong TFE3 expression and the preservation of TSC2 expression. These two sets of genes (TFE3, and TSC2/TSC1) have been suggested to be mutually exclusive and crucial in the pathogenesis of PEComas. 5

The TFE3 gene is located on the short arm of the $\mathrm{X}$ chromosome and normally encodes a transcription factor that binds one of the E-box sequences (present in the immunoglobulin enhancer) in the promoter sequences. ${ }^{9}$ Downstream of transforming growth factor beta signaling, TFE3 is promoted by encoded transcription factors. Xp11-associated renal cell carcinoma (now reclassified as the MiT family translocation renal cell carcinoma) and alveolar soft part sarcoma (ASPS) are associated with this translocation. TFE3 translocations may play regulatory roles in the epithelioid morphology of tumors arising from different origins. ${ }^{6}$

PR immunoreactivity in PEComa has also been described in a previous study. ${ }^{2}$ It has been suggested that female hormones play a role in the development of these tumors, considering their female predominance and the frequent involvement of gynecological organs. PEComa of the uterus described by Armah and Parwani ${ }^{2}$ showed strong positivity to PR and stronger positivity to ER. However, our case demonstrated PR positivity coupled with ER negativity. The significance of this finding has yet to be determined.

The malignancy criteria of PEComas have not been established because only extremely rare cases have metastasized or recurred. In one study of 26 PEComas, Folpe et al..$^{10}$ suggested that tumor size greater than $8 \mathrm{~cm}$, more than one mitosis per $50 \mathrm{HPFs}$, and presence of necrosis were strongly associated with malignant behavior. In another, Schoolmeester et al. ${ }^{11}$ suggested malignancy criteria intended only for gynecologic organs including tumor size greater than $5 \mathrm{~cm}$, high-grade nuclear features, necrosis, vascular invasion, and a mitotic rate greater than $1 / 50$ HPFs.

Although PEComas show a distinct morphology, other entities in the breast presenting an alveolar architecture of epithelioid cells should be considered in determining the identity. The differential diagnosis should include metastatic renal clear cell carcinoma, clear cell glycogen rich carcinoma, and ASPS as per histologic findings. Pancytokeratin negativity excludes the two carcinomas. While epithelioid nested architecture and TFE3 positivity are common histologic features of ASPS, HMB45 positivity excludes this diagnosis.

When pathologists encounter an unusual epithelioid tumor of the breast, PEComa should be considered in the differential diagnosis. In addition to HMB 45, other immunohistochemical data such as those of TFE3 and TSC2/TSC1 may be informative.

The accumulation of more data for PEComa and rigorous studies on relevant topics including genetic background may be helpful for determining the origin, prognosis, and treatment options for the rare cases of PEComa.

\section{ORCID}

Hyunjin Kim: https://orcid.org/0000-0001-8336-881X

Jimin Kim: https://orcid.org/0000-0002-3846-8093

Se Kyung Lee: https://orcid.org/0000-0003-1630-1783

Eun Yoon Cho: https://orcid.org/0000-0001-5849-3600

Soo Youn Cho: https://orcid.org/0000-0001-9714-7575

\section{Conflicts of Interest}

The authors declare that they have no potential conflicts of interest.

\section{REFERENCES}

1. Fletcher CD, Unni KK, Epstein J, Mertens F. World Health Organization classification of tumours: pathology and genetics of tumours of soft tissue and bone. Lyon: IARC Press, 2002; 221-2.

2. Armah HB, Parwani AV. Malignant perivascular epithelioid cell tumor (PEComa) of the uterus with late renal and pulmonary metastases: a case report with review of the literature. Diagn Pathol 2007; 2: 45 . 
3. Argani P, Aulmann S, Illei PB, et al. A distinctive subset of PEComas harbors TFE3 gene fusions. Am J Surg Pathol 2010; 34: 1395406.

4. Govender D, Sabaratnam RM, Essa AS. Clear cell 'sugar' tumor of the breast: another extrapulmonary site and review of the literature. Am J Surg Pathol 2002; 26: 670-5.

5. Malinowska I, Kwiatkowski DJ, Weiss S, Martignoni G, Netto G, Argani P. Perivascular epithelioid cell tumors (PEComas) harboring TFE3 gene rearrangements lack the TSC2 alterations characteristic of conventional PEComas: further evidence for a biological distinction. Am J Surg Pathol 2012; 36: 783-4.

6. Schoolmeester JK, Dao LN, Sukov WR, et al. TFE3 translocation-associated perivascular epithelioid cell neoplasm (PEComa) of the gynecologic tract: morphology, immunophenotype, differential diagnosis. Am J Surg Pathol 2015; 39: 394-404.

7. Martignoni G, Pea M, Reghellin D, Zamboni G, Bonetti F. PEComas: the past, the present and the future. Virchows Arch 2008; 452:
119-32.

8. Agaram NP, Sung YS, Zhang L, et al. Dichotomy of genetic abnormalities in PEComas with therapeutic implications. Am J Surg Pathol 2015; 39: 813-25.

9. NCBI. TFE3 transcription factor binding to IGHM enhancer 3 [Homo sapiens (human)] [Internet]. Bethesda: National Center for Biotechnology Information, 2018 [cited 2018 Aug 30]. Available from: https:/ / www.ncbi.nlm.nih.gov/gene?Db=gene\&Cmd=Deta ilsSearch\&Term $=7030$.

10. Folpe AL, Mentzel T, Lehr HA, Fisher C, Balzer BL, Weiss SW. Perivascular epithelioid cell neoplasms of soft tissue and gynecologic origin: a clinicopathologic study of 26 cases and review of the literature. Am J Surg Pathol 2005; 29: 1558-75.

11. Schoolmeester JK, Howitt BE, Hirsch MS, Dal Cin P, Quade BJ, Nucci MR. Perivascular epithelioid cell neoplasm (PEComa) of the gynecologic tract: clinicopathologic and immunohistochemical characterization of 16 cases. Am J Surg Pathol 2014; 38: 176-88. 